\title{
Differentiation of Atrial and Pulmonary Vein Potentials Recorded Circumferentially Within Pulmonary Veins
}

\author{
HIROSHI TADA, M.D., HAKAN ORAL, M.D., RADMIRA GREENSTEIN, M.D., \\ FRANK PELOSI JR., M.D., BRADLEY P. KNIGHT, M.D., \\ S. ADAM STRICKBERGER, M.D., and FRED MORADY, M.D.
}

From the Division of Cardiology, Department of Internal Medicine, University of Michigan Medical Center, Ann Arbor, Michigan

\begin{abstract}
Pulmonary Vein Electrograms. Introduction: Accurate discrimination of atrial and pulmonary vein potentials recorded circumferentially within the pulmonary veins is important when performing segmental isolation of the pulmonary veins in patients with atrial fibrillation.

Methods and Results: Twenty patients with paroxysmal atrial fibrillation underwent pulmonary vein mapping with a decapolar Lasso catheter during sinus rhythm and during pacing in the distal coronary sinus and left atrial appendage. Bipolar and unipolar electrograms were recorded within the left superior, right superior, and left inferior pulmonary veins. The atrial potentials were larger in the left pulmonary veins than in the right superior pulmonary vein, whereas the pulmonary vein potentials in the superior pulmonary veins were larger than in the left inferior pulmonary vein. The atrial and pulmonary vein potentials usually were readily distinguished during sinus rhythm in the right superior pulmonary vein. Characteristic distribution and morphologies of the atrial potentials as well as the response to distal coronary sinus and left atrial appendage pacing were useful for differentiating the atrial and pulmonary vein potentials in the left pulmonary veins.

Conclusion: Atrial and pulmonary vein potentials recorded circumferentially within the pulmonary veins have characteristic features that are useful in distinguishing them from each other. In the left pulmonary veins, discrimination of the atrial and pulmonary vein potentials is aided by coronary sinus or left atrial appendage pacing. (J Cardiovasc Electrophysiol, Vol. 13, pp. 118-123, February 2002)
\end{abstract}

\section{pulmonary vein, atrial fibrillation, atrial potential, pulmonary vein potential}

\section{Introduction}

Segmental isolation of the pulmonary veins to eliminate atrial fibrillation may be guided by pulmonary vein potentials recorded circumferentially near the ostia of the veins. ${ }^{1,2}$ However, atrial potentials also are recorded by electrodes positioned within the pulmonary veins, and accurate differentiation of the atrial and pulmonary vein potentials at times may be difficult. The aim of this study was to analyze the characteristics of the electrograms recorded circumferentially within pulmonary veins that allow atrial and pulmonary vein potentials to be distinguished.

\section{Methods}

\section{Patient Characteristics}

The subjects of this study were 20 patients ( 17 men and 3 women; mean age $47 \pm 14$ years) with drug-refractory, paroxysmal atrial fibrillation who underwent segmental isolation of the pulmonary veins. Mean duration of symptomatic atrial fibrillation was $4.5 \pm 4.8$ years, and the patients experienced a mean of $14 \pm 11$ episodes/month. One patient

Supported in part by a grant from the Gunma Prefecture Government and by the Don Nouse Arrhythmia Research Fund.

Address for correspondence: Fred Morady, M.D., Division of Cardiology, Department of Internal Medicine, University of Michigan Medical Center, 1500 E. Medical Center Drive, B1F245, Ann Arbor, MI 48109-0022. Fax: 734-936-7026; E-mail: fmorady@ umich.edu

Manuscript received 12 September 2001; Accepted for publication 18 December 2001. had hypertension; the remaining 19 patients had no structural heart disease. Echocardiography demonstrated a mean left ventricular ejection fraction of $0.60 \pm 0.05$ (range 0.50 to 0.70 ) and a mean left atrial diameter of $38 \pm 5 \mathrm{~mm}$ (range 32 to 45$)$.

\section{Electrophysiologic Study Protocol}

Therapy with antiarrhythmic drugs was discontinued at least 48 hours before the procedure, except in the case of amiodarone, which was discontinued at least 6 weeks before the procedure. After informed consent was obtained, a quadripolar electrode catheter (EP Technologies Inc., San Jose, CA, USA) was inserted into the right femoral vein and positioned in the distal portion of the coronary sinus. Transseptal catheterization was performed under guidance of intracardiac echocardiography. A 7-French electrode catheter with $101-\mathrm{mm}$ electrodes spaced at intervals of 4.5 or $6.0 \mathrm{~mm}$ in a distal ring configuration (Lasso ${ }^{\mathrm{TM}}$; Biosense Webster, Inc., Diamond Bar, CA, USA) was inserted into the left atrium. Heparin was administered as needed to maintain an activated clotting time of 250 to 350 seconds.

Surface leads and intracardiac electrograms were recorded digitally (EP Med Systems, Inc., Mount Arlington, NJ, USA). The filter settings were 30 to $500 \mathrm{~Hz}$ for bipolar electrograms and 0.05 to $200 \mathrm{~Hz}$ for unipolar electrograms. An indifferent electrode positioned on the right thigh was used for unipolar recordings. Pacing was performed with a stimulator (model EP-3 Clinical Stimulator; EPMed Systems) at twice diastolic threshold and with a pulse width of $2 \mathrm{msec}$. 
Figure 1. Bipolar recordings near the ostium of the left superior pulmonary vein (A) and right superior pulmonary vein $(B)$. Shown are lead II, electrograms recorded by the Lasso catheter, and the stimulus (S) channel, during sinus rhythm and coronary sinus (CS) pacing. Coronary sinus pacing makes the pulmonary vein potentials (arrows) stand out from the atrial potentials in the left superior pulmonary vein (A), but causes fusion of the atrial and pulmonary vein potentials in the right superior pulmonary vein. Pulmonary vein potentials always were validated by their disappearance after radiofrequency ablation (RFA), as shown in the panel on the left.

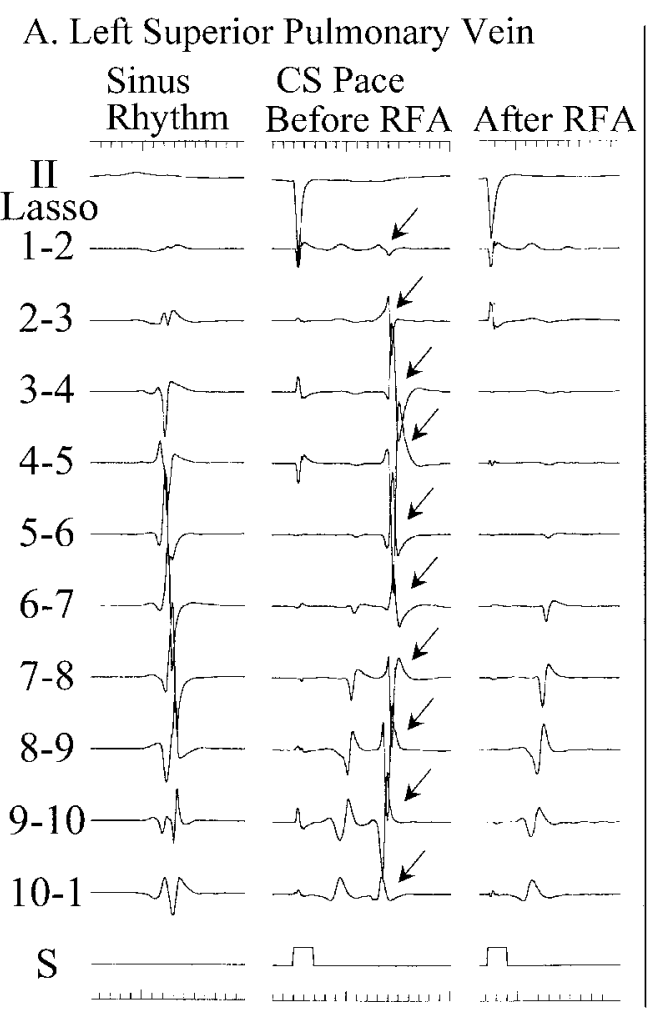

\section{Study Protocol}

Pulmonary vein angiograms were performed to identify the location of the ostia. The Lasso catheter was positioned sequentially within the left superior, left inferior, and right superior pulmonary veins, within $5 \mathrm{~mm}$ of the ostium. Electrograms recorded within the right inferior pulmonary vein were not included in this study because the Lasso catheter usually could not be positioned within this vein. Ten bipolar electrograms recorded by the adjacent pairs of electrodes and unipolar electrograms recorded by each of the 10 electrodes of the Lasso catheter were recorded during sinus rhythm and during distal coronary sinus pacing at a cycle length of $600 \mathrm{msec}$. Coronary sinus pacing was performed for optimal separation of atrial and pulmonary vein potentials. ${ }^{2}$ In 12 patients, the bipolar and unipolar electrograms recorded near the ostia of the left superior pulmonary vein also were recorded during pacing from the left atrial appendage.

\section{Analysis of Electrograms}

Pulmonary vein potentials were defined as deflections that had a sharp upstroke, duration $<50 \mathrm{msec}$, amplitude $>0.05 \mathrm{mV}$, and followed the atrial potential..$^{1,3}$ Correct identification of pulmonary vein potentials was validated by their disappearance after radiofrequency ablation. Atrial potentials were not affected by radiofrequency energy applications (Fig. 1A).

Measurements of electrode width, amplitude, and timing were performed with electronic calipers. Bipolar electrograms were analyzed for amplitude and width of the pulmonary vein and atrial potentials (Fig. 2). The widths of the atrial and pulmonary vein potentials were measured at the Lasso catheter recording site that displayed the largest elec-
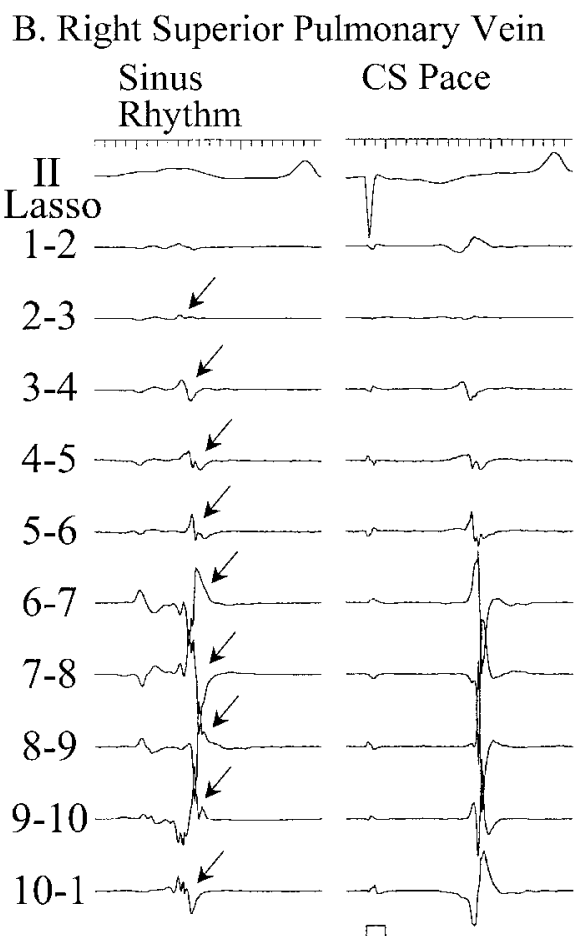

$\mathrm{S}$ $100 \mathrm{~ms}$ trogram amplitudes. The maximum electrogram width was defined as the interval from the earliest deflection of the atrial potential to the termination of the pulmonary vein potential. To be considered an electrogram deflection, a potential had to have an amplitude $\geq 0.05 \mathrm{mV}$. The separation or overlap between the atrial and pulmonary vein potentials was evaluated during sinus rhythm and distal coronary sinus pacing (Figs. 1 and 3 ).

Unipolar electrograms recorded by the Lasso catheter were analyzed for the initial polarity of the atrial potential (Fig. 4). The positive and negative slopes of the atrial and pulmonary vein potentials also were measured.

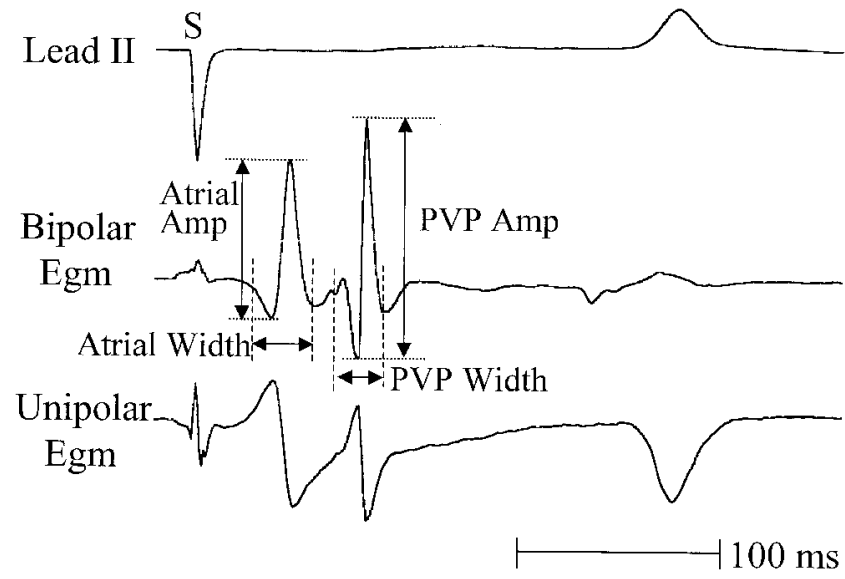

Figure 2. Bipolar and unipolar recordings at a proximal site in the left superior pulmonary vein. Shown are lead II, a bipolar electrogram, and a unipolar electrogram recorded by the Lasso catheter. Amp = amplitude; Egm = electrogram $; P V P=$ pulmonary vein potential; $S=$ pacing stimulus. 


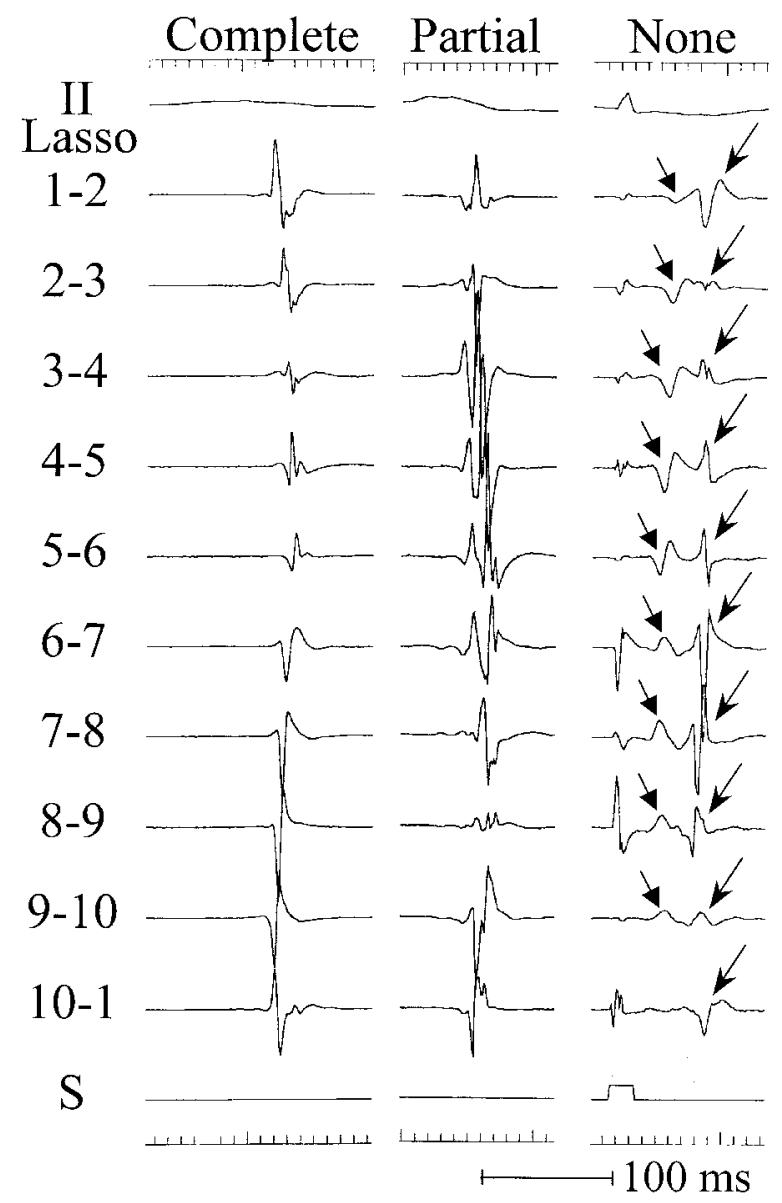

Figure 3. Examples of varying degrees of overlap of the atrial and pulmonary vein potentials. Shown are lead II, bipolar electrograms recorded by the Lasso catheter, and the stimulus (S) channel.

\section{Statistical Analysis}

Continuous variables are expressed as mean $\pm 1 \mathrm{SD}$ and compared using analysis of variance (ANOVA). When group differences were found, one-way ANOVA was followed by the Tukey-Kramer method to test the significance of the difference among means in all groups. Categorical values were compared by Chi-square analysis. $\mathrm{P}<0.05$ was considered statistically significant.

\section{Results}

\section{Overlap of Atrial and Pulmonary Vein Potentials}

In bipolar recordings, the atrial and pulmonary vein potentials overlapped in $97 \%$ of the left-sided pulmonary veins during sinus rhythm. During coronary sinus pacing, the maximum electrogram width increased significantly (Table 1). The percentage of left-sided pulmonary veins in which bipolar electrograms demonstrated overlap of the atrial and pulmonary vein potentials decreased from $97 \%$ during sinus rhythm to $64 \%$ during coronary sinus pacing $(\mathrm{P}<0.001)$.

In $35 \%$ of right superior pulmonary veins, the atrial and pulmonary vein potentials overlapped during sinus rhythm. During coronary sinus pacing, the maximum electrogram width remained unchanged or became shorter in $55 \%$ of right superior pulmonary veins (Table 1 ). The percentage of right superior pulmonary veins in which bipolar electrograms demonstrated overlap of the atrial and pulmonary vein potentials increased from $35 \%$ during sinus rhythm to $95 \%$ during coronary sinus pacing $(\mathrm{P}<0.001)$.

\section{Initial Polarity of the Atrial Potential}

In unipolar recordings, the atrial electrogram in all pulmonary veins was a slow deflection. During sinus rhythm, the initial polarity of the atrial potential was positive in the left superior and inferior pulmonary veins (Fig. 4A) and negative in the right superior pulmonary vein (Table 1 and Fig. 4B). During coronary sinus pacing, the initial deflection of the atrial potential remained positive in the left pulmonary veins (Fig. 4A) and changed from negative to positive in $95 \%$ of right superior pulmonary veins $(\mathrm{P}<0.001$; Table 1 and Fig. 4B).

\section{Amplitudes and Widths of the Atrial and Pulmonary Vein Potentials}

The maximum amplitude of the atrial potential was greater in the left pulmonary veins than in the right superior pulmonary vein in bipolar recordings $(\mathrm{P}<0.001$; Fig. 5A). The number of electrode pairs of the Lasso catheter that recorded an atrial amplitude $>0.2 \mathrm{mV}$ in bipolar recordings was $4.8 \pm 1.8$ of 10 in the left superior pulmonary vein and $3.8 \pm 2.2$ of 10 in the left inferior pulmonary vein versus $1.4 \pm 2.0$ pairs of 10 in the right superior pulmonary vein $(\mathrm{P}<0.001)$.

The maximum amplitude of the pulmonary vein potential was greater in the left and right superior pulmonary veins than in the left inferior vein in bipolar recordings $(\mathrm{P}<0.05$; Fig. 5A). In bipolar recordings, the maximum amplitude of the atrial potential was significantly smaller than that of the pulmonary vein potential in both superior pulmonary veins (P $<0.001$; Fig. 5A).

In both left pulmonary veins, the atrial potentials were significantly wider than the pulmonary vein potentials $(\mathrm{P}<$ 0.001; Fig. 5B).

\section{Morphology of Atrial and Pulmonary Vein Potentials}

In unipolar recordings, the atrial potential had a $\mathrm{QS}$ or $\mathrm{rS}$ pattern in the right superior pulmonary vein during sinus rhythm and an RS pattern in both left pulmonary veins during coronary sinus pacing (Fig. 4). The pulmonary vein potential had an RS or $\mathrm{rS}$ pattern in all pulmonary veins (Fig. 4). The positive and negative slopes of the pulmonary vein potential were significantly greater than those of the atrial electrograms ( $\mathrm{P}<0.001$; Fig. 6$)$. In both left pulmonary veins, the atrial potentials had a $\mathrm{QR}$ or rSR pattern in bipolar recordings (Fig. 7).

\section{Left Atrial Appendage Pacing}

The percentage of left-sided pulmonary veins in which bipolar electrograms demonstrated overlap of the atrial and pulmonary vein potentials decreased from $64 \%$ during coronary sinus pacing to $17 \%$ during left atrial appendage pacing ( $\mathrm{P}<0.01$; Fig. 8). 
Figure 4. Unipolar recordings near the ostium of the left inferior pulmonary vein $(A)$ and right superior pulmonary vein (B). Shown are lead II, 10 electrograms recorded by the Lasso catheter, and the stimulus (S) channel, during sinus rhythm (left) and coronary sinus (CS) pacing (right). Note that the initial polarity of the atrial potential is positive in the left inferior pulmonary veins during sinus rhythm and CS pacing (A). In the right superior pulmonary veins $(B)$, the initial polarity of the atrial potential was negative during sinus rhythm but changed from negative to positive during CS pacing.

\section{A. Left Inferior Pulmonary Vein}

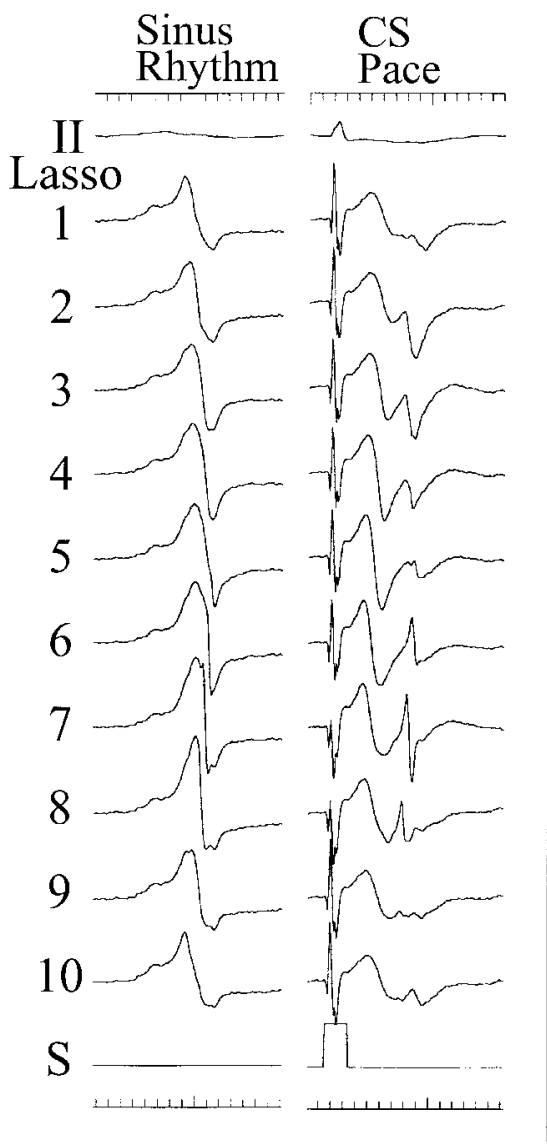

B. Right Superior Pulmonary Vein

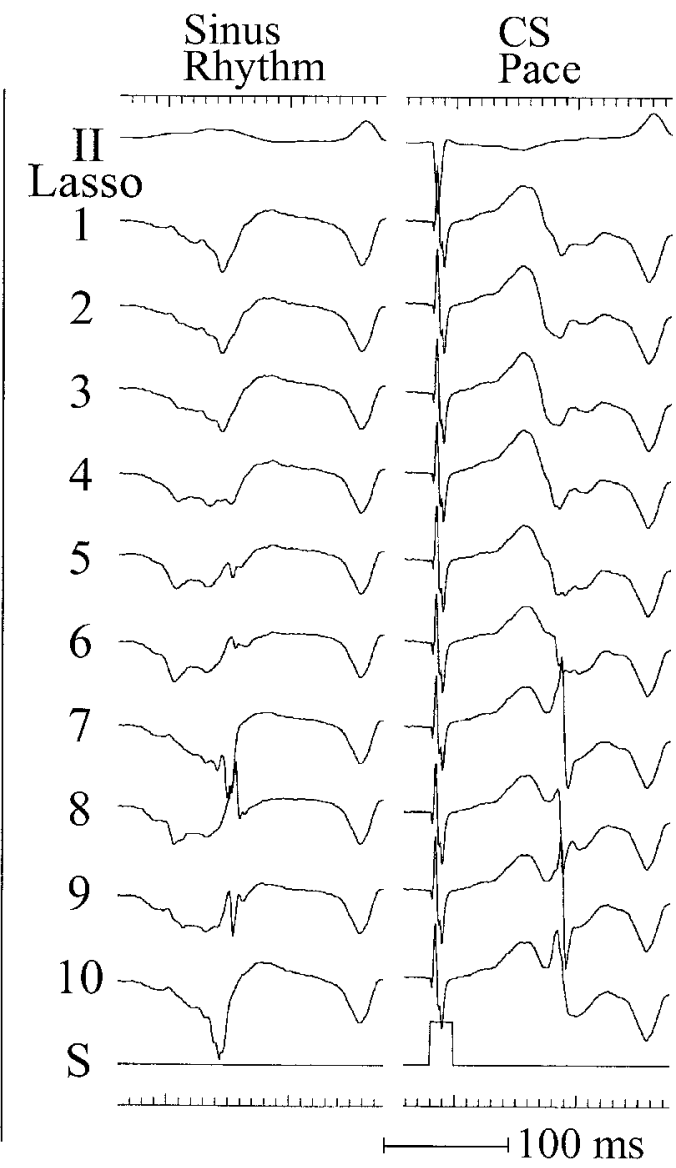

\section{Discussion}

\section{Differentiation of Atrial and Pulmonary Vein Potentials}

In the right superior pulmonary vein, the atrial potentials are much smaller than the pulmonary vein potentials, and there is little overlap during sinus rhythm. Therefore, it usually is not difficult to distinguish the atrial and pulmonary vein potentials in this vein.

On the other hand, in the left superior and inferior pulmonary veins, distal coronary sinus pacing is needed to separate the atrial from the pulmonary vein potential. In these two veins, the atrial and pulmonary vein potentials may have similar amplitudes; in $65 \%$, there is overlap of the two potentials even during coronary sinus pacing. In this event, pacing from the left atrial appendage is helpful in advancing the atrial potential and eliminating overlap with the pulmonary vein potentials. These prominent atrial potentials, which are mainly recorded at the anterior aspect of the left superior and inferior pulmonary veins, may reflect activation of the left atrial appendage, which lies in close proximity and anterior to the left pulmonary veins. ${ }^{4,5}$

In addition to their characteristic distribution along the anterior aspect of the veins, ${ }^{5}$ the atrial potentials recorded within the left superior and inferior pulmonary veins have other distinguishing features. These atrial potentials are wider than the pulmonary vein potentials, have an initial

TABLE 1

Changes of Measurement Variables During Sinus Rhythm and During Coronary Sinus Pacing

\begin{tabular}{|c|c|c|c|c|c|c|}
\hline \multirow[b]{2}{*}{ Variables } & \multicolumn{2}{|c|}{ LSPV } & \multicolumn{2}{|c|}{ RSPV } & \multicolumn{2}{|c|}{ LIPV } \\
\hline & SR & CS Pace & SR & $\overline{\text { CS Pace }}$ & SR & $\overline{\text { CS Pace }}$ \\
\hline Maximum Egm width (msec) & $34 \pm 10$ & $56 \pm 17 \dagger \uparrow$ & $38 \pm 13$ & $38 \pm 11$ & $34 \pm 13$ & $47 \pm 17 *$ \\
\hline Initial polarity of atrial potential & & & & & & \\
\hline Flat & 0 & 2 & 1 & 1 & 1 & 1 \\
\hline Negative & 0 & 0 & 19 & 0 & 0 & 0 \\
\hline
\end{tabular}

Values are given as mean $\pm \mathrm{SD}$.

$* \mathrm{P}<0.05 ; \dagger \mathrm{P}<0.01$ vs SR; $\$ \mathrm{P}<0.001$ vs CS Pace in RSPV.

$\mathrm{CS}=$ coronary sinus; $\mathrm{Egm}=$ electrogram; LIPV = left inferior pulmonary vein; LSPV = left superior pulmonary vein; PVP = pulmonary vein potential; $\mathrm{RSPV}=$ right superior pulmonary vein; $\mathrm{SR}=$ sinus rhythm. 


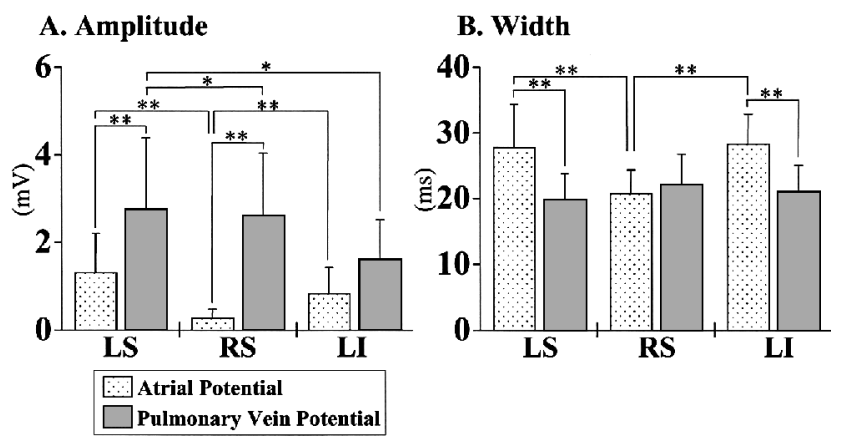

Figure 5. Measurements of bipolar electrograms at the pulmonary vein ostia. (A) Maximum amplitude of the atrial and pulmonary vein potentials. (B) Width of the atrial and pulmonary vein potentials. Values are expressed as mean \pm 1 SD. These measurements were obtained during coronary sinus pacing for left-sided pulmonary veins and during sinus rhythm for the right superior pulmonary veins. $L I=$ left inferior pulmonary vein; $L S=$ left superior pulmonary vein; $R S=$ right superior pulmonary vein. $* P<$ $0.05 ; * * P<0.001$.

negative polarity in bipolar electrograms, and have slower upstrokes and downstrokes than the pulmonary vein potentials in unipolar electrograms.

\section{Atrial Potentials Recorded in the Left Pulmonary Veins}

An activation wavefront resulting from pacing in the distal coronary sinus would be expected to travel from the inferolateral aspect of the left atrium in a rightward and superior direction toward the left atrial appendage. The left atrial appendage is located anterior and superior to the left pulmonary veins.4,5 Therefore, atrial potentials recorded within the two pulmonary veins might be expected to be most prominent along the anterior aspects of the veins. The direction of the activation wavefront relative to the Lasso catheter electrodes near the ostia of the left pulmonary veins may explain the negative initial polarity of the atrial potentials, with a QR or rSR pattern in the bipolar recordings at the anterior aspect of these veins.

\section{Amplitude of Pulmonary Vein Potentials}

The muscle sleeves that surround the superior pulmonary veins are larger than those that surround the inferior pul-

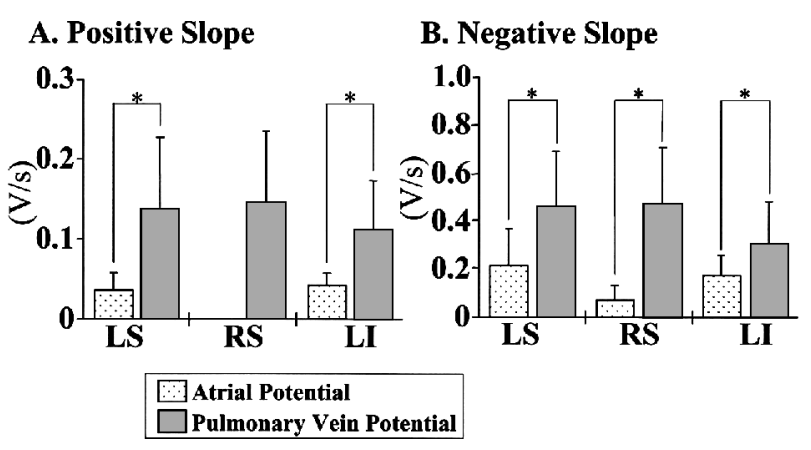

Figure 6. Measurements of unipolar electrograms recorded at the pulmonary vein ostia. (A) Positive slope of the potentials. (B) Negative slope of the potentials. Values are expressed as mean $\pm 1 S D . L I=$ left inferior pulmonary vein; $L S=$ left superior pulmonary vein; $R S=$ right superior pulmonary vein. $* P<0.001$.

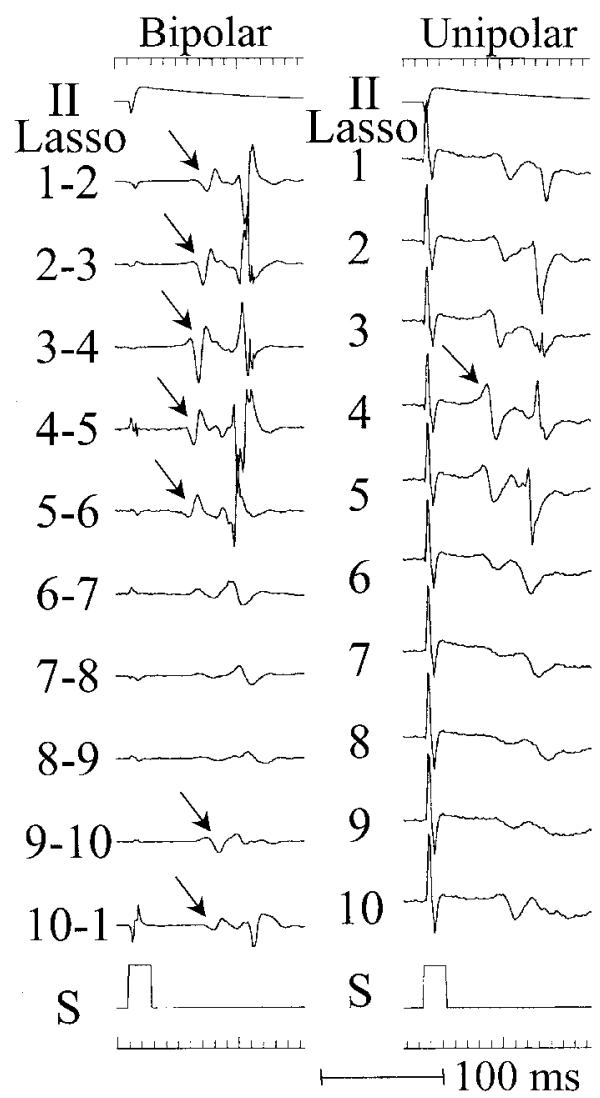

Figure 7. Bipolar and unipolar recordings near the ostium of the left superior pulmonary vein during coronary sinus pacing. Shown are lead II, 10 electrograms recorded by the Lasso catheter, and the stimulus (S) channel. In the bipolar recordings, atrial potentials (arrows) have an initial negative polarity, with a $Q R$ or rSR pattern. The unipolar electrogram recorded at site 4 has the maximum amplitude, with a RS pattern (arrow), and the positive and negative slopes are less steep than those of the pulmonary vein potential.

monary veins. ${ }^{4,6,7}$ In this study, the pulmonary vein potentials recorded in the left and right superior pulmonary veins were larger than those recorded in the left inferior pulmonary vein, suggesting the muscle sleeves surrounding the superior pulmonary veins also may be thicker.

\section{Study Limitations}

One limitation of this study is that there was no control group of subjects without atrial fibrillation. Therefore, the extent to which the characteristics of pulmonary vein electrograms described in this study are specific to patients with atrial fibrillation is unknown. A second limitation is that circumferential electrograms recorded from within the right inferior pulmonary vein were not analyzed in this study because of difficulty in positioning the Lasso catheter within this vein. Finally, the 10 electrodes of the Lasso catheter all may not have always been in good contact with the wall of the pulmonary vein, and this may have affected the characteristics of the electrograms that were analyzed in this study. Furthermore, the electrogram characteristics may have been affected by the position of the Lasso catheter in the pulmonary veins. For this reason, care was taken to always position the Lasso catheter within $5 \mathrm{~mm}$ of the ostium of the pulmonary veins. 


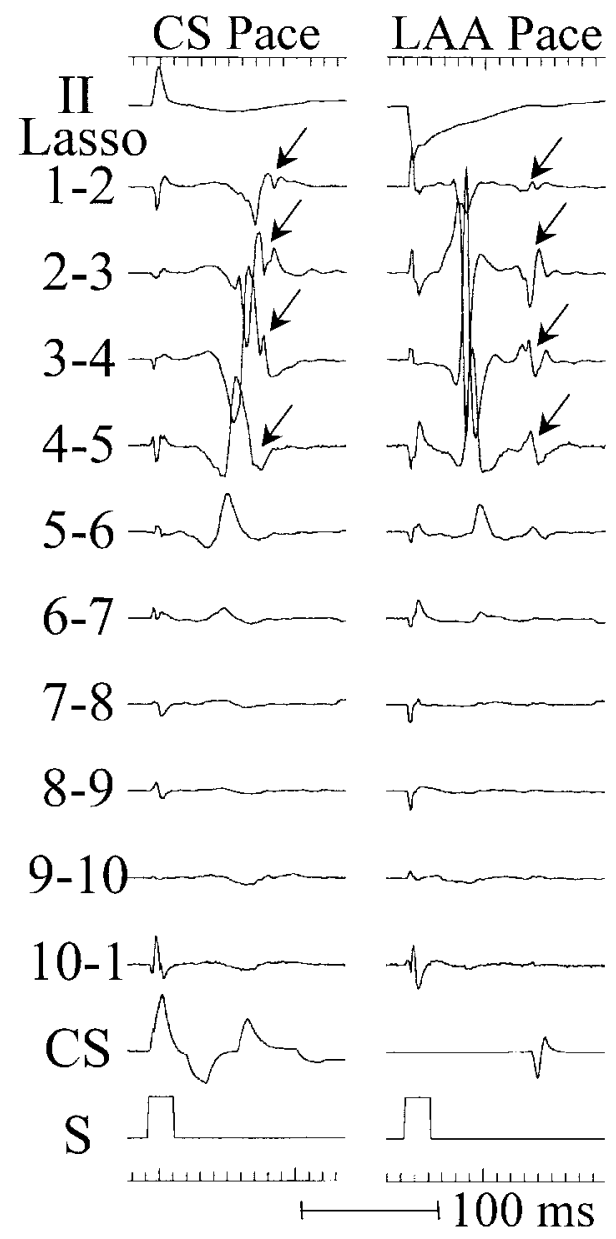

Figure 8. Bipolar recordings near the ostium of the left inferior pulmonary vein during pacing from the distal coronary sinus $(C S)$ and from the left atrial appendage (LAA). Shown are lead II, 10 electrograms recorded by the Lasso catheter, an electrogram recorded in the CS, and the stimulus (S) channel. The pulmonary vein potentials (arrows) are clearly separated from the atrial potentials during pacing from the LAA but not during CS pacing.

\section{Clinical Implications}

An endpoint of segmental isolation of the pulmonary veins is the elimination of all pulmonary vein potentials at the ostia of the pulmonary veins. Because left atrial myocardium and the left atrial appendage are in close proximity to the ostia of the pulmonary veins, the electrograms recorded circumferentially from within the pulmonary veins, particularly the left superior and inferior pulmonary veins, often contain a prominent atrial component. To achieve successful isolation of a pulmonary vein with a minimum number of applications of radiofrequency energy and to be able to recognize when complete isolation has been achieved, accurate discrimination of atrial and pulmonary vein potentials is essential. The findings of this study demonstrate that the atrial and pulmonary vein potentials recorded circumferentially within the pulmonary veins can be distinguished during sinus rhythm in the right pulmonary vein and during either distal coronary sinus or left atrial appendage pacing in the left superior and inferior pulmonary veins.

\section{References}

1. Haissaguerre M, Shah DC, Jais P, Hocini M, Yamane T, Deisenhofer I, Chauvin M, Garrigue S, Clementy J: Electrophysiological breakthroughs from the left atrium to the pulmonary veins. Circulation 2000;102:2463-2465

2. Hocini M, Shah DC, Jais P, Haissaguerre M, Peng JT, Yamane T, Deisenhofer I, Garrigue S, Clementy J: Concealed left pulmonary vein potentials unmasked by left atrial stimulation. PACE 2000;23(Pt II): 1832-1835

3. Chen SA, Hsieh MH, Tai CT, Tsai CF, Prakash VS, Yu WC, Hsu TL, Ding YA, Chang MS: Initiation of atrial fibrillation by ectopic beats originating from the pulmonary veins: Electrophysiological characteristics, pharmacological responses, and effects of radiofrequency ablation. Circulation 1999;100:1879-1886

4. Ho SY, Sanchez-Quintana D, Cabrera JA, Anderson RH: Anatomy of the left atrium: Implication for radiofrequency ablation of atrial fibrillation. J Cardiovasc Electrophysiol 2000;11:758-761.

5. Shah D, Haissaguerre M, Jais P, Hocini M, Teiichi Y, Deisenhofer I, Choi KJ, Macle L, Clementy J: Left atrial appendage potentials masquerade as left superior pulmonary vein activity. (Abstract) PACE 2001;24(Pt II):541.

6. Nathan $\mathrm{H}$, Eliakim M: The junction between the left atrium and the pulmonary veins. An anatomic study of human hearts. Circulation 1966;34:412-422.

7. Saito T, Waki K, Becker A: Left atrial myocardial extension onto pulmonary veins in humans: Anatomic observations relevant for atrial arrhythmias. J Cardiovasc Electrophysiol 2000;11:888-894. 